\title{
Polyvinylidene Difluoride Piezoelectric Electrospun Nanofibers: Review in Synthesis, Fabrication, Characterizations, and Applications
}

\author{
Zainab Abu Alhasssan, ${ }^{1}$ Yasmeen S. Burezq, ${ }^{1}$ Remya Nair, ${ }^{1}$ and Nader Shehata $\mathbb{D}^{1,2,3,4}$ \\ ${ }^{1}$ Kuwait College of Science and Technology (KCST), Doha District, P. O. Box 27235, Safat 13133, Kuwait \\ ${ }^{2}$ Department of Engineering Mathematics and Physics, Faculty of Engineering, Alexandria University, Alexandria 21544, Egypt \\ ${ }^{3}$ Center of Smart Nanotechnology and Photonics (CSNP), SmartCI Research Center, Alexandria University, Alexandria 21544, Egypt \\ ${ }^{4}$ Faculty of Science, Utah State University, Logan, Utah 84322, USA
}

Correspondence should be addressed to Nader Shehata; nader.shehata@alexu.edu.eg

Received 25 January 2018; Revised 29 July 2018; Accepted 4 October 2018; Published 4 December 2018

Academic Editor: Bhanu P. Singh

Copyright (C) 2018 Zainab Abu Alhasssan et al. This is an open access article distributed under the Creative Commons Attribution License, which permits unrestricted use, distribution, and reproduction in any medium, provided the original work is properly cited.

\begin{abstract}
This review article highlights the methods and principles used for PVDF nanofiber creation and the use of these nanofibers in different fields by utilizing its piezoelectric performance, etc. The studies include different techniques to improve the alignment and piezoelectric behavior of nanofibers. The pressure versus sensitivity behavior of PVDF substrate is thoroughly analyzed. The optimum conditions (of experimental parameters) of the electrospinning technique are carefully studied to improve the structure, alignment, and performance of the generated nanofiber mats. In addition to experimental methods, the preparation of nanocomposites with carbon nanotubes (CNTs) also can improve the structure, alignment, and piezoelectric performance. The characterization tools used for the analyses of fabricated nanofibers are also discussed, in addition, to analyze the piezoelectric results of the targeted nanocomposite.
\end{abstract}

\section{Introduction}

Nanotechnology is a distinctive branch of science and engineering dealing with matter on a molecular or even atomic scale. The study of materials in nanoscale which is one billionth of a second allows us to exploit most of the properties exhibited by matters in nanoscale. A wide variety of nanostructures are available nowadays, and it mainly includes nanorods, nanofibers, nanoparticles, nanowires, and nanotubes. Among these, nanofibers are characterized by several fascinating properties like large surface to volume ratio, high porosity, good mechanical performances, and flexibility in surface functionality that captivate our field of interest. When thousands of nanofibers are created close to each other using some special techniques, it results in the formation of nanosheets or mats and a clear picture about the size of nanofibers can be obtained by comparing it with the size of bacteria. It is not possible for the bacteria to penetrate through these sheets since the pore space available is less than the size of bacteria, thus nanofiber mats can be used for protection against bacteria and viruses, and at the same time it allows air molecules to pass through it. Such benefits can be utilized in the preparation of nanofibrous breathing mask which allows comfortable germ-free breathing by blocking the entry of viruses and bacteria [1-3].

A variety of natural, synthetic, ceramic, or composite polymers are used in different geometric shapes ranging from yarn, nonwoven web, and bulk structures for the production of nanofibers. As a result, nanofibers with different physical properties and application potentials can be produced. The main reason behind their selection for nanofiber fabrication is their presence of functional group, elasticity, flexibility, and springiness. The organic polymers are mainly insulators in nature, but some intrinsically conducting polymers are also available in nature which is 
having alternate double and triple bond along the polymer backbone. These conducting polymers are made of aromatic rings naphthalene, thiophene, etc., which are connected to one another through carbon single bonds. Polyacetylene or polyetheneis the first synthesized polymer, and thefirst attempt did not yield a very good conductive polymer, and the conductivity was significantly improved by oxidizing it with halogen vapors. This conductive polymer yielded the high conductivity compared toallotherpolymersknownat that time. Natural polymers mainly include collagens, cellulose, keratin, and polysaccharides, and the synthetic polymers mainly used for nanofiber generation include polylacticacid (PLC), nylon 6, polyvinyl fluoride (PVF), polyvinyl chloride (PVC), and polyurethane (PU). Most of these polymers are used for fabrication of nanofibers in solution form by mixing with appropriate solvents. The most commonly used solvents include formic acid, dimethylformamide (DMF), distilled water, and acetone. Different types of nanofibers can be fabricated by varying the concentration of solution and also by changing the solvents and solutes in appropriate manner. The mainfactorsaffected by concentrationinclude viscosity, surface tension, and tensile strength. As a result, the fabricated nanofibers will be of different diameters and softness. Sometimes, even fiber structure or morphology is also affected resulting in the formation of nanobeads, helical nanofibers, and hollow fibers [4].

Our special case of interest focuses on polyvinylidene fluoride (PVDF) for generating nanofibers because of its unique electroactive properties such as piezo-, pyro-, and ferroelectric properties. PVDF is a special chemically inert thermoplastic fluoropolymer synthesized by the polymerization of vinylidene fluoride. It also exhibits several useful properties such as lightweight, flexibility, high purity, resistance to solvents, and stability under high electric field. PVDF's piezoelectric property can be utilized for several advanced applications of nanofibers including energy harvesters, force sensors, and transducers. Its ability to produce large voltages with low forces makes it much favorable to use in piezoelectricity. The polar crystalline nature of PVDF is the main reason for its superior piezoelectric performance. The molecular structure of PVDF consists of repeated monomer units such as $(-\mathrm{CH} 2-\mathrm{CF} 2-) \mathrm{n}$ with a crystallinity of $50 \%$ such that the crystallites are embedded in amorphous domain and a low melting point of 177 degree Celsius. Based on the chain conformation (order of polymer chains in unit cell) as trans or gauche linkages, PVDF exists in four different crystalline forms such as alpha, beta, gamma, and delta phases. Among this, the only polymorph which exhibits piezoelectric nature is beta phase with a strong dipole moment. It possesses an oriented hydrogen and fluoride unit cells along with the carbon backbone, and thus it becomes useful in most of the applications of nanofibers. But the most abundant form of PVDF available is its alpha phase. So, the nonpolar alpha phase can be converted to polar beta phase by the application of high electric field and stretching $[5,6]$.

The most important property of PVDF which is utilized to the maximum in the fabrication of nanofibers is its piezoelectric property. There are many fibers with piezoelectric properties such as PZT, PVC, nylon 11, and PVDF [7-9]. But due to PVDF properties and its ability to produce large voltages with low forces, it makes them much favorable to use in piezoelectricity. The phenomenon of linear electromechanical interaction between the mechanical and electrical state in crystalline material with no inversion symmetry is called piezoelectric effect. The effect is reversible in nature. Thus, an internal electrical charge can be developed by applying a mechanical stress and also an applied external electric field can generate an internal mechanical stress. Piezoelectricity was first discovered by the Curie brothers in 1880. One of the main parameters that decide the piezoelectric performance of a sample is its piezoelectric coefficient $\left(D_{33}\right)$ value. It quantifies the deformation or volume change occurred when a piezoelectric material is placed in an electric field or it can be defined as the ratio of polarization to the applied stress. Usually, piezoelectric coefficients are of two types: (a) piezoelectric charge constant denoted by " $d$ " and piezoelectric voltage constant denoted by " $g$." Piezoelectric charge coefficient gives the polarization generated per unit mechanical stress, and voltage coefficient gives the electric field generated by the piezoelectric material per unit mechanical stress applied. The material suitability for strain-dependent applications can be easily identified by knowing its $D_{33}$ value. Generally, the coefficient is labeled as $D_{x y}$ and its number is based on the direction of polarization and stress direction. The coefficient value of $D_{33}$ is the one taken into account in our studies for the piezoelectric behavior analysis [10].

In a piezoelectric material, the piezoelectric behavior is experienced only in a range of temperature which is below a particular critical temperature called Curie temperature; above this temperature, each unit cell of the material exhibits a cubic symmetry with no dipole moment where it is in a paraelastic phase. Below Curie temperature, the unit cell exhibits a tetragonal or rhombohedral symmetry leading to a dipole moment resulting in a ferroelectric phase. Generally, a piezoelectric material has a randomly aligned electrical domain. When an electric field is applied to a piezoelectric material, as it passes close to Curie temperature, spontaneous polarization is developed such that all the polarization vectors are aligned in a more or less uniform direction. This process by which a net polarization is developed is called poling. So it will exhibit piezoelectric property only after the process of electrical poling where the electrical domains are aligned resulting in a dipole moment and creation of polarization. The process of poling consists of inducing a DC voltage across the material. By the application of pressure on a piezoelectric material, the negative charges are deposited on the expanded side and positive charges are deposited on the compressed sides. Thus, when the pressure is relieved, electric current starts to flow across the material. This effect has a wide range of application such as production and detection of sound, high voltage generation, and frequency generation. Piezoelectricity is the basis of several scientific instruments such as STM and AFM [11].

A wide range of experimental techniques are available for the production of nanofibers in both small scale and large scale. The techniques mainly include template synthesis, thermal-induced phase separation, self-assembly, interfacial 
polymerization, solution blowing or air jet spinning, drawing techniques, freeze or drying synthesis, melt spinning and stretching, spinneret-based tunable engineered parameter (STEP) method, force spinning or centrifugal spinning, electrospinning, and mechanical spinning. Among these, our field of interest mainly focuses on the fabrication of PVDF nanofibers; the main techniques used nowadays specially for PVDF fibers are electrospinning, melt spinning and stretching, centrifugal spinning, and mechanical spinning. Melt spinning and stretching method is mainly used for the production of nanofiber membranes with high mechanical strength. Centrifugal spinning or force spinning is an alternative method useful for the fabrication of PVDF nanofibers where centrifugal forces are used for the production of fibers. Here, a syringe pump or an extruder is used, and the polymer solution is fed through it to a rotating chamber having two or more orifices. The centrifugal force pushes the polymer solution towards the center of the chamber where there are orifices during the time of rotation. When the centrifugal force dominates the surface tension and viscosity of the solution, it escapes from the orifices and then the jet elongates and solution evaporates until it reaches the collector plate where it is deposited. Electrospinning, the most versatile and promising technique, uses electrostatic force instead of centrifugal force for the fabrication of PVDF fibers [9-12].

The basic principle behind electrospinning technique is the uniaxial stretching of a polymer solution which is viscoelastic by applying electrostatic forces. A continuous set of nanofibers can be generated without any disruption if we have enough solution to feed. Well-aligned and random nanofibers can be generated by altering some of the experimental parameter values of electrospinning. Here, a high DC voltage is applied to the needle of a syringe where the solution is fed using a precision pump and a rotating drum or stationary plate is used as collector for depositing the fiber. The same experimental setup is also used for mechanical spinning in the absence of the electrical bias, and instead of electrical bias, fiber drawing was maintained by an appropriate match between the feeding rate of solution supplying system and rotary speed of a drum. Most of the techniques discussed here encountered several limitations including lack of control over generated fiber diameter and its orientation and low production rate. But compared to other techniques, electrospinning is a very successful method in the creation of nanofibers with controllable parameters. Most of the useful properties of the PVDF nanofibers can be enhanced by optimizing the experimental parameters of electrospinning in a controlled fashion $[6,13]$.

\section{Experimental Background}

2.1. The Experimental Setup. The experimental setup of electrospinning mainly includes a high voltage power supply (DC of the order of several kilovolts), injection pump, metallic syringe with a needle of inner diameter of the order of $\mathrm{mm}$, and a collector electrode which is a stationary plate or a rotational drum. The initial step involved in the experimental procedure is turning on the power supply, and then the prepared PVDF polymer solutions are forced through the metallic syringe. As a result, a drop of the polymer solution is formed at the tip of the needle. But since the needle is under the effect of a high voltage supply, charges are induced within the fluid. As a result, the surface of the ejected drop distorts into the shape of a cone known as Taylor cone. The arrangements are clearly shown in the block diagram shown in Figure 1.

When a high voltage is applied into the polymer solution, it induces free charges; this makes the system get a positive charge and the collector electrode (a flat plate or rotating drum) get a negative charge. Consequently, an electric field is created. This electric field is created between the tip of the needle and the collector. The reason for using high voltage supply is to produce electrically charged jets from polymer solution or melts, which on drying by means of evaporation of the solvent produce PVDF fibers. Here, when the electrostatic force becomes dominant over the surface tension of the liquid droplet, the droplet will get distorted which forms the so called Taylor cone. This distortion leads to electricity charge (current) resulting in a jet ejection moving towards the collector, which forms the aligned or unaligned PVDF fiber $[14,15]$.

Electrospinning jet ejected from the needle under the influence of the high voltage takes three forms: in the first region just after ejection, solution drops form a cone shape called Taylor cone but it takes only a small portion approximately around $1 \mathrm{~mm}$; then from the bottom of the cone, it travels straight like a thin line linearly around $1-2 \mathrm{~mm}$ which is called the stability region; and finally the fluid line region or jet is disturbed resulting in a bending instability region where jet bends and whips erratically due to the high electrostatic force experienced. This unstable region is the lengthiest region which is approximately around $5-10 \mathrm{~cm}$ which finally gets coated on the collector electrode in the form of a random nonwoven mat. The reduction of the diameter of the fluid line happens at the final instability region resulting in fine nanofibers. Therefore, the main area where we focus our studies is the bending instability part [16-18].

\subsection{Variable Parameters in the Experimental Part}

2.2.1. Selection of the Source Solution with Required Concentration. Our special case of interest focuses on PVDF solution for the fabrication of nanofibers. But based on the solvent and solute concentration, the features of output nanofibers vary. The solvent mainly used for PVDF solution preparation is DMF/acetone, N-methy1-2-pyrrolidinone (NMP), N,N-dimethylacetamide/acetone, etc.; here, the solvent is mixed with the available PVDF powder. The solution is prepared by varying the molecular weight of the powdered PVDF added to the solvent and also by varying the solvent concentration. This has an important role in creating nanofibers with different diameters and alignments (random or aligned) [19].

As the concentration changes from very low to high, the generated fibers are of different morphologies. If the concentration is too low, polymeric micro- or nanoparticles are formed and the technique undergone here will be electrospraying instead of electrospinning due to high surface 


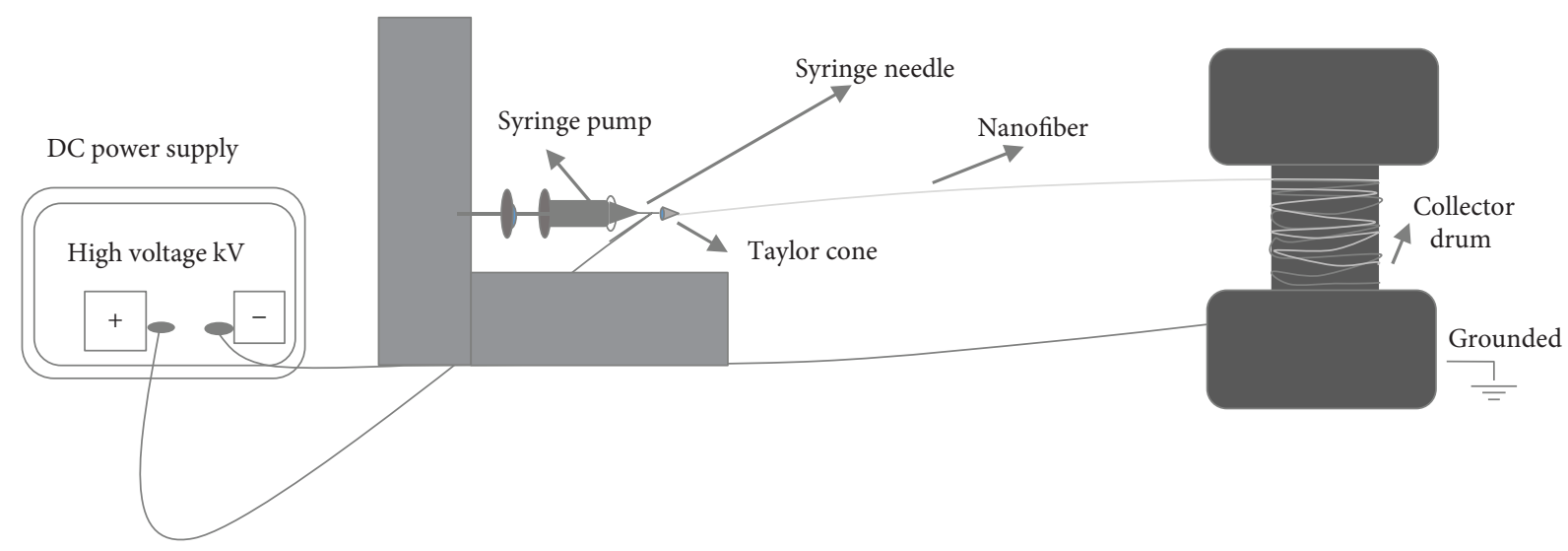

FIGURE 1: Electrospinning setup with a drum.

tension and low viscosity at this condition. When the concentration is increased a little, a mixture of beads and fibers is formed and the process will be electrospinning. By increasing the concentration a little and finally when it reaches the optimum concentration, smooth nanofibers will be obtained. If we increase the concentration further, the resultant will be helix-shaped microscaled ribbons instead of nanoscaled fibers. A small increase in the concentration within the optimum range results in the increase in diameter of smooth fibers. Studies done by Zhu et al. showed that a change in concentration from 8 to $14 \%$ of PVDF solution resulted in a fiber diameter variation from $20 \mathrm{~nm}$ to $100 \mathrm{~nm}$, as shown in Figure $2[13,19]$.

An important parameter to be taken into account to enhance the piezoelectric property of the PVDF nanofiber is poling electric field, poling time, and poling temperature. These parameters have to be utilized to a maximum for getting an optimum aligned beta sheets of nanofiber which can be used for energy scavenging applications. The spontaneous polarization of beta sheets of PVDF can be utilized for many applications; thus, the parameters have to be controlled well for generating a good piezoelectric nanofiber.

The piezoelectric behavior of PVDF nanofibers can be improved by addition of carbon nanomaterials such as carbon allotrope fullerenes (C60) and single- and multiwalled carbon nanotubes. These additives can improve the crystalline nature and electrical conductivity thereby increasing the optimum performance of PVDF nanofibers.

2.2.2. Selection of Solution Delivery System. The solution delivery system which is mainly the needle design has a great influence on the structure of output nanofiber. Different types of needle designs available are single-needle nozzle, multineedle nozzle, coaxial spinneret, and bicomponent spinneret, and also in some cases, needless electrospinning setups can be used, such that the fabricated nanofibers with different morphologies and structures can be developed. In the single-needle setup, the solution is delivered through the single needle; in contrast, needless setup includes a solution feeder from a widely opened surface which results in large nanobeads also with the generated nanofibers. Another needless technique involves a

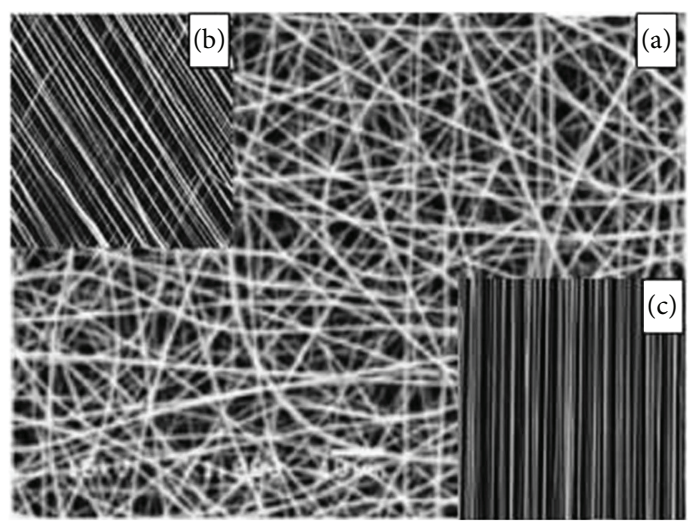

FIgURE 2: An example of characterization of electron spun nanofibers using SEM: (a) random NFs, (b) aligned fibers at an angle, and (c) aligned fibers kept straight [19].

rotating roller partially immersed into the polymer solution resulting in conical spikes on the solution layer. Different types of rotating roller shapes are also available such as disc, cylinder, or ball which can greatly influence the structure and morphology of fiber. Another type of needless spinneret available is spiral coil wire-type design. Thus, a variety of needless source delivery systems including open space, rotating roller, and spiral coil wire and different types of singleneedle systems such as coaxial and bicomponent spinnerets are available through which we can create fibers of required demand by controlling the above parameters $[20,21]$.

(1) Initial Diameter of the Needle. The initial diameter of the needle is also a control parameter in this technique since the decrease in needle diameter changes the amount of solution drop coming out of the needle. For example, if the needle diameter is in the range of millimeters, a needle diameter of $0.25 \mathrm{~mm}$ is used in their studies. When the size of the droplet decreases, surface tension increases resulting in a decreased acceleration of the jet so that it takes a long time for the jet to reach the collector electrode. At the same time, if the needle diameter is too small, it cannot allow the droplet to escape from the needle tip [22-24]. 
2.2.3. Viscosity of Polymer Solution. Viscosity of the applied polymer solution plays an important role in the fabrication of fibers. When a polymer of higher molecular weight is added to the solvent, it increases the viscosity. Highly viscous fluid cannot be transferred through the needle tip. At the same time, very low viscous fluids can result in generation of beads; furthermore, an increase in viscosity gradually changes the shape of beads from spherical to spindle until a smooth fiber is obtained. So a medium viscosity which is required for the optimum design of fibers showed that a change in concentration from 8 to 14 wt.\% of PVDF solution resulted in a viscosity variation from $304.2 \mathrm{mPa}$ to $1845.3 \mathrm{mPa}$ [15].

2.2.4. Conductivity of Solution. Conductivity of a solution mainly depends on polymer type, solvent sort, and the salt used. Conductivity of the polymer solution can be changed by the addition of ions by adding small amount of salt or polyelectrolyte to the solution. When conductivity increases, it results in the increase of charge concentration carried out by the jet thereby increasing the stretching of jet resulting in more smooth fibers. Zhu et al. showed that a change in polymer solution from 8 to $14 \mathrm{wt} . \%$ resulted in conductivity

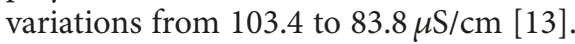

2.2.5. Feed Rate or Flow Rate. The rate at which the drops are fed out also plays an important role in the fabrication of fibers. To maintain a stable Taylor cone, there is a standard feed rate for each applied voltage. Increase in the feed rate $(0.40$ and $0.66 \mathrm{ml} / \mathrm{h})$ affects the fiber diameter or bead size. Thus, with the increase in feed rate, there will be an increase in fiber diameter. If the feed rate is too high, fibers with beads are formed; the reason is that there is no enough time for solvent evaporation [25].

2.2.6. Distance from Needle Tip to Collector. The parameter that gets affected by changing the distance between the needle tip and collector (in range of $10-15 \mathrm{~cm}$ ) is the flight time and field strength. Depending on the distance between rotating collector electrode and the polymer solution source, electrospinning technique is divided into two types: near-field electrospinning process (NFES) since the order of $1 \mathrm{kV}$ and far-field electrospinning process (FFES). In NFES, the needle tip to collector distance is adjusted to be in the range of millimeters $(\mathrm{mm})$ and the applied voltage is of the order of $1 \mathrm{kV}$. Here, the short distance between the source and collector helps us to utilize the stable liquid jet region that can result in a controlled sequential distribution of the fibers on the collector. This is possible because of the large electric field in the small gap between the needle and collector electrode. Thus, in NFES, the fiber deposition can be done in a more controllable manner, such that the fibers can be distributed in an ordered fashion on the collector electrode $[10,16,26]$.

In FEFS, the needle tip to collector distance is adjusted to be in the range of the order of centimeters particularly above ten centimeters. As a result, the stability of the liquid jet will be affected and it undergoes a chaotic whipping process resulting in a randomly oriented fiber deposition on the collector electrode. In FFES, the applied voltage is in the range of ten kilovolts, and the source needle diameter is of the order of around 100 micrometers. But in this case, the alignment can be improved by choosing fast spinning electrodes in the form of rotating drum, wheel-like disk, and also wire drums $[10,16,26]$.

By reducing or increasing the applied voltage, the stretching of the solution droplet and electrostatic force can be affected resulting in changes of the fiber structure. The output parameter that gets greatly changed by the influence of voltage is the fiber diameter, but the effect of voltage is greatly depending on two other factors such as polymer solution concentration and the distance between the tip and collector electrode [10].

2.2.7. Effect and Shape of Collector Electrode. Different types of collector electrodes include stationary conductive metallic sheet and rotating drum. Also, the electrodes can be conductive or nonconducting; if it is conductive, then a stable potential difference can be maintained between the source and collector. But if the collector electrodes are nonconducting, charges on the jet will accumulate on the collector electrode resulting in less deposit of fibers. Thus, a lower packing density of fibers is obtained with nonconducting electrodes compared to conducting electrodes where fibers are closely packed. Also, the stationary and rotating collectors play an important role in the alignment of fibers; a perfectly aligned nanofibers is difficult to synthesize and it can be made practical using fast spinning electrode such as rotating drum, wheel-like disk, grids, parallel or gridded bar, and also wire drum collector. In the case of a stationary plate electrode, random or messy fibers will be created [27].

2.2.8. Surface Tension. Surface tension is an important factor affecting the fiber nature. During the pathway between Taylor cone and collector plate, the surface tension of the fluid has to be reduced for yielding more uniform fibers. The surface tension can be reduced by adding more surfactants, and addition of solvent ethanol can greatly reduce surface tension so that more uniform fibers can be obtained. With the concentration fixed, reducing the surface tension of the solution, beaded fibers can be converted into smooth fibers. Surface tension and viscosity can be adjusted by changing the mass ratio of the solvent mix if there are two solvents. In some research works, there was a variation of surface tension from $46 \mathrm{mN} / \mathrm{m}$ to $30 \mathrm{mN} / \mathrm{m}$ when the ratio of ethanol to DMF was varied from $100 / 0$ to $0 / 100$, respectively [28].

2.2.9. Temperature Treatment or Thermal Energy. The microstructure and mechanical and piezoelectric properties of electrospun nanofibers can be greatly altered by temperature treatment or annealing which is an ambient parameter. The increase in temperature favors the production of thinner nanofibers than that created at low temperatures. When the temperature was varied from $30^{\circ} \mathrm{C}$ to $60^{\circ} \mathrm{C}$, the fiber diameter was varied from $98 \mathrm{~nm}$ to $90 \mathrm{~nm}[14,20,29]$.

2.2.10. Humidity. Humidity is another important ambient parameter that affects the morphology of the fabricated fiber. 
As humidity increases, the drying time increases resulting in the variation of the diameter of the generated fiber [30].

\subsection{Analytical Instruments Used for the Characterization of} Fabricated Fibers. The surface morphology of the nanofibers is characterized by using a scanning electron microscope (SEM). The diameter of the generated fibers can also be determined using SEM. In some cases where there is requirement to analyze the bead formation in fabricated fiber, atomic force microscopy (AFM) is also used. More detailed structural investigation of a single nanofiber is also done by using transmission electron microscopy (TEM). The infrared spectra of the fiber mats are recorded using Fourier-transform infrared spectrometer (FTIR). Based on the sensitivity of CF2 orientation changes, FTIR characterizes both the dipole orientation and crystallographic structure of nanofibers. The crystalline structural analysis of the fabricated fibers is done using X-ray diffractometer (XRD). It clearly depicts the presence of beta phase which is responsible for piezoelectric behavior in a nanofiber.

The piezoelectric performance which can be characterized based on the output voltage generated by the applied stress can be studied using a piezoelectric force microscope (PFM). It allows local piezoelectric measurements, and so it is best suited for the characterization of individual nanofiber mats. Simple harmonic generation is another characterization technique for studying polarization behavior. Raman spectroscopy can be used for the detection of low-frequency mode in a system.

I-V characteristics of the generated sample can be studied using a Keithley source measurement unit (four-point probe). The resistivity and I-V characteristics of the generated sample can be studied using this technique. The change in microstructure and electromechanical and piezoelectric behavior of the electrospun nanofiber due to annealing treatment is studied using PFM, DSC, XRD, FTIR, and AFM nanoindentation [20,30-33].

\section{Results and Discussion}

Electrospinning is the most promising technique for the fabrication of nanofibers in a controllable manner. Using this method, nanofibers of diameter ranging from ten nanometers to several orders of micrometers can be fabricated. This technique is relatively simple, fast, reliable, and efficient compared to other similar techniques. The main principle behind this technique or one of the crucial factors affecting this technique is the electric field applied between the source and collector electrode. The changing electric field profile between the source and collector greatly influenced the diameter and alignment of generated fiber.

In addition to fiber diameter, morphological variations in fibers can be obtained by changing some of the experimental parameters of electrospinning setup. Different types of fibers fabricated by modifying the technique slightly are branched split nanofibers, porous nanofiber, flattened ribbon-like fiber, helical nanofiber, and hollow nanofiber. Split fibers can be fabricated by the separation of a primary jet into two smaller jets. Similarly, flattened ribbons like nanofiber were created with higher molecular weight and higher polymer concentration. Here, since the viscosity of solution is high reduction of water, solvent evaporation takes place and as a result, due to the high impact, the wet fibers that reach the fiber collector are flattened. Here, first the tube collapses due to high pressure and the circular cross section initially became elliptical shaped and then into a ribbon-like shape. Based on the polymer source solution mixture, solvent evaporation results in a thermodynamically unstable stage, which leads to two phases. The polymer-rich phase which is of gel type solidifies rapidly after phase separation and forms the base which is the matrix, and the polymerdeficient phase forms the pores. Another important favorable factor for porous nanofiber is the humidity of electrospinning environment. Also, bicomponent nanofiber with different decompositions can be fabricated using two capillaries placed side by side within a single capillary such that the resultant fiber is having dual components.

3.1. Morphology and Diameter. The morphology and diameter of the electrospun nanofibers are studied mainly using a scanning electron microscope; the morphology mainly including random or messy and aligned or ordered arrangement of nanofibers can be easily identified using this technique. The same technique can be used for diameter analysis of fabricated fiber synthesized by varying different experimental parameters. An example of characterization of nanofibers using SEM is depicted.

\subsubsection{Variations in Electrospinning Technique Parameters for} Optimizing the Structure and Morphology of Fabricated Fiber. The diameter of the fabricated nanofibers can be varied by varying the distance between the source needle and collector electrode and also by controlling the applied voltage. Generally, NFES produces a controlled number of aligned PVDF nanofibers and even a single fiber can be fabricated at a time. But FFES generally produces random or messy fibers with large population. Here, the alignment or the proper order of the fibers can be improved by changing the collector electrode to a rotating drum or disk. The diameter of the fibers generated will be higher in the case of NFES and lesser in the case of FFES. NFES of PVDF resulted in a fabricated fiber of diameter in the range $500-6500 \mathrm{~nm}$ in the case of a single fiber and a diameter range of $1000-2000 \mathrm{~nm}$ in the case of multifiber generation. At the same time, FFES produced a single fiber with a diameter range of $600 \mathrm{~nm}$ and multifiber with a diameter range of $187 \mathrm{~nm}[10,34]$.

Another important parameter affecting the morphology and diameter of nanofibers is the rotating speed of the collector electrode. The stretching rate of the polymer jet is increased by increasing the rotating speed, and as a result, the increased rate of rotation results in a narrower distribution of fibers. Thus, thin fibers are fabricated by increasing the speed of rotation and thick fibers are formed with slower rate of rotation. With a constant applied voltage and source to collector distance, the rotation per minute is varied from 500 to 2000 in increments of $500 \mathrm{rpm}$ and the fiber diameters become narrower. Another notable property observed 
is that the alignment is found to be increased and more orderly arranged fibers are obtained [30].

Liu et al. studied the effect of fiber diameter on rotational velocity of collector electrode by fixing the applied voltage, $x y$ platform control speed, and PVDF solution concentration at $16 \mathrm{kV}, 2 \mathrm{~mm} / \mathrm{s}$, and $18 \mathrm{wt} \%$, respectively, using hollow cylindrical near-field electrospinning technique (HCNFES). Studies concluded that the rotational speed is inversely proportional to fiber diameter. The average fiber diameter ranges from $100 \mathrm{~nm}$ to 1 micrometer. Here, the rotational velocity selected to fabricate PVDF fiber was 900 to $1900 \mathrm{rpm}(900,1100,1300,1500,1700$, and 1900) which resulted in a good and continuous fibers with decreasing diameters up to $100 \mathrm{~nm}$ from 1.2 micrometer as shown in Figure 3. Here, a medium rotational speed is selected because under low rotational speed, the speed with which fibers are deposited exceeds the rotational speed of collector resulting in a random disorganized and disordered collection on nanofibers. At the same time, a very high rotational speed cannot be selected since it can result in a deposition of continuous broken fibers, because the generated fibers can be mechanically broken due to the high speed which is not an optimum condition for well-aligned PVDF fibers through electrospinning. Also, the effect of various compositions of PVDF polymer solution is important on the fabricated nanofiber. The composition mainly included DMSO (dimethly sulfoxide) and acetone as a solvent and Zonyl as fluorosurfactant. The solvents are fixed at a ratio of $1: 1$, and the surfactant weight was fixed to be $0.2 \mathrm{~g}$, then the weight percentage of the PVDF powder with solvent is varied from 16 wt. $\%$ to $18 \mathrm{wt} . \%$ and $20 \mathrm{wt} . \%$. These varying concentrations are produced for characterization of surface tension and viscosity of the polymer solution in HCNFES process. The detailed investigation of the fabricated fibers in all these cases revealed that lower weight concentration below $16 \mathrm{wt} . \%$ resulted in a discontinuous and nonuniform fiber diameters. The studies revealed that $18 \mathrm{wt} . \%$ concentration is the most optimum condition for synthesizing good electrospun nanofibers. Relating to voltage dependence for this, the PVDF solution concentration, the $x y$ platform speed, and the rotational speed of the collector are fixed at $18 \mathrm{wt} . \%, 2 \mathrm{~mm} / \mathrm{s}$, and $1500 \mathrm{rpm}$, respectively. Applied DC voltage is varied from $10 \mathrm{kV}$ to $16 \mathrm{kV}$, and the corresponding average diameters are obtained in the range from 1.16 micrometer to $200 \mathrm{~nm}$, respectively, which is shown below in Figure 3 [20].

\subsubsection{Varying the PVDF Solution Content for Enhanced Performance}

(1) Addition of Different Solvents to the Solution with Different Solvent Concentrations. Generally, variations in PVDF solution contents are done for fabricating the most efficient fiber. The studies using conventional electrospinning method mainly used a solution made from 18 weight percent PVDF dissolved in DMF and acetone in a 3:1 volume to volume ratio with the inclusion of light-emitting polymer, $\operatorname{poly}(9,9-$ dioctylfluorene-alt-benzothiadiazole) (F8BT), as well as 16 weight percent PVDF dissolved in DMF. For preparing a uniform solution, PVDF was always added first followed by DMF and then finally acetone if it was required for preparation. If there is any addition of fluorophore, it has to be dissolved first in one of the solvents and thoroughly mixed and then added to the PVDF. A hot plate heated to a temperature of $50^{\circ} \mathrm{C}$ is used to keep both solutions and is stirred carefully for an hour before being used for electrospinning. This can allow the PVDF to dissolve completely so that a homogeneous solution is prepared for electrospinning. Here, the solution having $18 \mathrm{wt} . \%$ is delivered through the source needle with a needle to collector distance of $10 \mathrm{~cm}$ with an applied voltage of $13 \mathrm{kV}$ and a flow rate of $0.5 \mathrm{~mm} / \mathrm{hr}$. For $16 \mathrm{wt} . \%$ solution, the source to collector distance is set to $15 \mathrm{~cm}$ with an applied voltage of $15 \mathrm{kV}$ at a flow rate of $0.7 \mathrm{~mm} / \mathrm{hr}$. The generated nanofibers in both cases are analyzed under SEM. Only samples that form stable jet are used for investigation. By the thorough analyses of a set sample created with each solution, it is concluded that the average diameter of the fiber is $200 \mathrm{~nm}$ with a standard deviation of $110 \mathrm{~nm}$ for the solution with fluorophore and the average diameter of the fiber is found to be $180 \mathrm{~nm}$ with a standard deviation of $118 \mathrm{~nm}$ for $16 \mathrm{wt} . \%$ solution, with a rotation speed of $140 \mathrm{rpm}$. Some others used the same technique and same solution with a rotating speed of $550 \mathrm{rpm}$. The needle to collector distance, appropriate solvent choice, and applied voltage can be modified to reduce the average diameter obtained in both cases [20].

Also, the concentration or molecular weight percent of PVDF together with the solvent affects the piezoelectric performance or beta-phase content. For the analysis of betaphase content, as shown in Figure 4, X-ray diffractometer (XRD) is used. The characterization is done by analyzing the prominent diffraction peaks of alpha-non-polar phase and beta polar phase in each case. The XRD characterization of the pure PVDF powder and electrospun fibers fabricated with 16 wt. $\%, 18$ wt.\%, and 20 wt.\% PVDF solution is done. For each of these cases, the applied voltages are varied in the order from $10,12,14$, and $16 \mathrm{kV}$ and the corresponding beta-phase peaks are analyzed. The XRD analyses of pure PVDF powder showed diffraction peaks at angle 2 theta degrees 17.9 (100), 18.4 (020), 20.1 (110), and 26.7 (021). The corresponding miller indices of each angle are shown in brackets. Among these, the only diffraction peak at 20.1 with miller indices 110 corresponds to the beta phase which is polar and all other phases are alpha which are nonpolar. By the addition of $16 \mathrm{wt} . \%$ and $18 \mathrm{wt} . \%$ with different applied voltages, it is found that most of the alpha polar phases disappeared and the diffraction peak that corresponds to beta phase with miller indices 110 became prominent and the intensity is found to be more with 10 close $\mathrm{kV}$ and then decreasing orderly from 10 to $16 \mathrm{kV}$. But it is observed that for a higher concentration up to $20 \mathrm{wt} . \%$, which is close to equilibrium solubility limit, the intensity of beta-phase peaks is found to be decreased. This is because here it is difficult to achieve homogeneous solution. Based on all these studies, in order to obtain a smooth surface morphology, high structural density, and good piezoelectric performance, the optimal parameters are decided to be the PVDF solution combination 


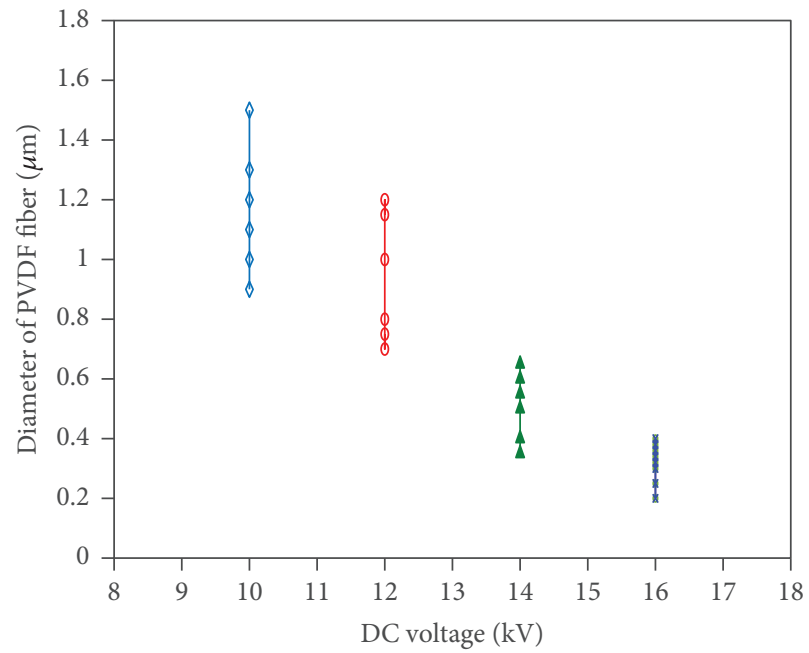

(a)

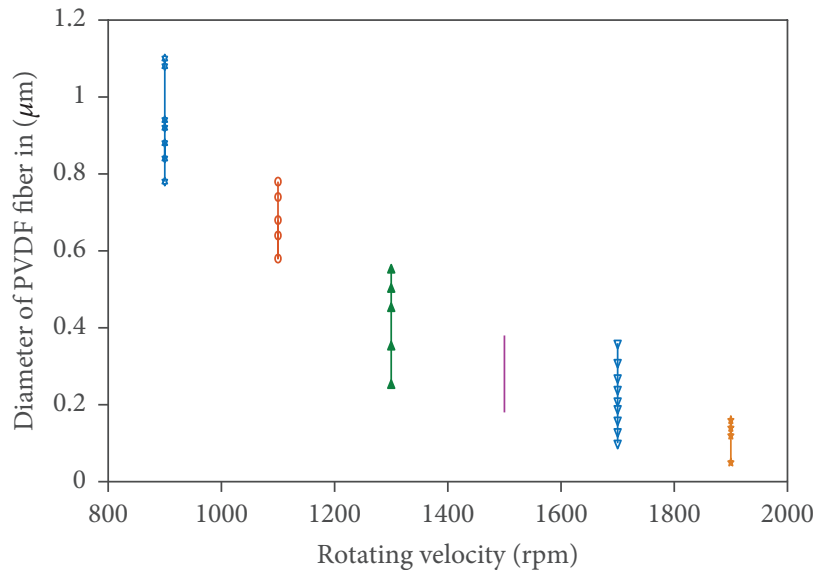

(b)

Figure 3: (a) Applied DC voltage versus the diameter of HCNFES PVDF fibers (the variance of the data point is $~ 2 \%-4 \%$ ); (b) rotation velocity versus the diameter of HCNFES PVDF fibers (the variance of the data points is $\sim 2-4 \%$ ).

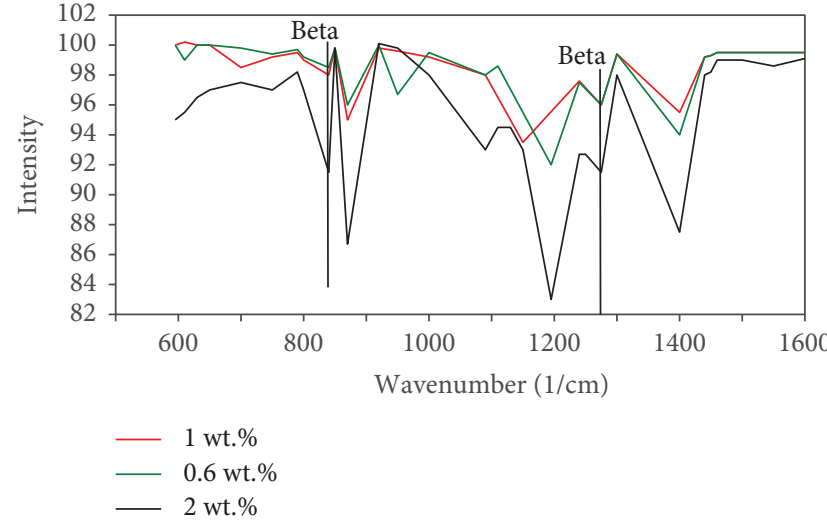

(a)

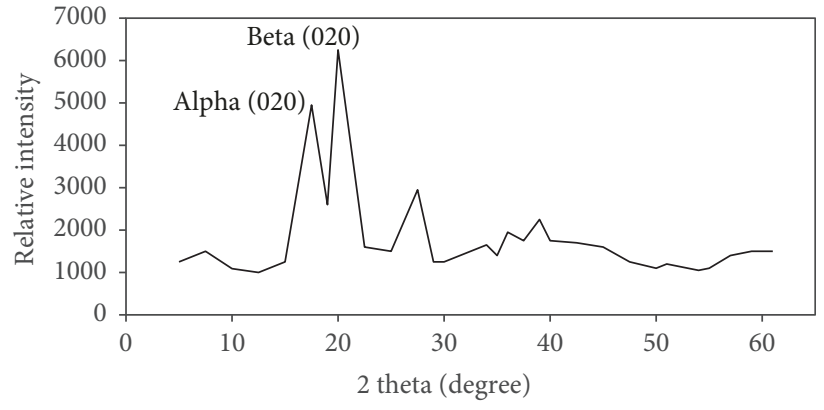

(b)

FIGURE 4: FTIR and XRD diffraction patterns of the aligned electrospun PVDF/MWCNT fibers. (a) FTIR spectra of some aligned electrospun PVDF/MWCNT fibers with MWCNT 0.6 and $1.2 \mathrm{wt} \%$ (b) XRD diffraction pattern of the aligned electrospun PVDF/MWCNT fibers with MWCNTs of $2 \mathrm{wt} \%$ [44].

18 wt.\% with a DC voltage of $14 \mathrm{kV}$ and rotational speed of $1900 \mathrm{rpm}$ [20, 35-38].

(2) Effect of Inorganic Salt on PVDF Solution. There are other studies that have found that the addition of inorganic salts on PVDF solution results in a decrease of surface tension and viscosity of the solution and at the same time increase in the conductivity. As a result, ultrathin fine fibers are fabricated. As we increased the concentration of $\mathrm{NaCl}$ from 0 to $0.6 \mathrm{wt} . \%$, the diameter of the electrospun nanofiber diameter is found to be reduced from $99 \mathrm{~nm}$ to $80 \mathrm{~nm}[39,40]$.

3.2. Piezoelectric Performance of the Fabricated Fiber Mat. The pressure versus voltage which is the sensitivity graph of a fabricated fiber (electrospun at a rate of $1500 \mathrm{rpm}$ ) is studied for its piezoelectric performance. After a continuous pressure of $0.145 \mathrm{MPa}$ and a frequency of $0.66 \mathrm{~Hz}$ were applied, the output response was very high with a big difference. With increasing pressure frequency, the output voltage is found to be not increasing much until it reaches a value of $0.145 \mathrm{MPa}$ and then it is found that there is an abrupt increase in output voltage. This is because of electrical poling in electrospinning process, which can also play another role for the dipoles oriented in a preferential direction. When electrospun fiber mats were deformed due to pressure input, the charges would be generated from the mat surface because of the unbalanced redistribution within the mats. Therefore, increasing pressure magnitude or frequency will cause more charges distributed on the surface, such that a higher output voltage is developed. The fabricated electrospun PVDF fiber mat showed a sensitivity of $178 \mathrm{mV} / \mathrm{kPa}$. But when we increased the frequency gradually, by keeping 
the pressure at a constant value of $0.145 \mathrm{MPa}$, the voltage generated is found to be almost constant with a slight change or increase in voltage level $[6,41,42]$.

3.3. Piezoelectric Behavior of a Fabricated Nanofiber. The most important fascinating property of the PVDF fiber fabricated using electrospinning technique is its piezoelectric performance. The piezoelectric behavior can be improved in a controlled rate via electrospinning technique. This property by which mechanical energy can be transformed to electrical energy can be utilized for several applications ranging from sensors to power generation. PVDF shows excellent piezoelectric performance and so it can be used for strain sensing applications.

The FTIR spectra of four different fabricated aligned electrospun nanofibers are analyzed by keeping the experimental parameters such as PVDF solution concentration to be $16 \mathrm{wt}$ \% with an applied voltage of $5 \mathrm{kV}$ and tip to collector distance of $10 \mathrm{~cm}$. Here, the parameter that was changed was the rotary speed of the collector electrode; it was varied by 500 , 1000,1500 , and $2000 \mathrm{rpm}$. The FTIR spectra showed some featured peaks at bands of $475,510,1275$, and $1431 \mathrm{~cm}^{-1}$, and all these bands correspond to the polar beta phase of PVDF which clearly indicates that aligned electrospun nanofibers with rich beta phase contents can be generated by optimizing the experimental parameters in a controlled manner [6].

Several additives are added to PVDF source solutions to increase the piezoelectric performance in electrospinning technique. Hydrated salt, nickel chloride hexahydrate $(\mathrm{NiCl} 2 \cdot 6 \mathrm{H} 2 \mathrm{O})$, is added to the PVDF solution for increasing the polar beta phase by about 30\% which leads to 3 times increase of peak to peak piezovoltage generation compared to electrospun pure PVDF. This increase in performance was attributed to ionic interaction between salt and polymer molecules. But at $\mathrm{NiCl} 2 \cdot 6 \mathrm{H} 2 \mathrm{O}$ concentration greater than 0.5 wt. $\%$, the piezoelectric response starts to drop due to the excess water content in the fiber [43].

3.3.1. Addition of Carbon Nanotubes (S/M) to PVDF for Its Improved Performance. One important additive of PVDF solution is the multiwalled carbon nanotubes (CNTs) to produce a nanocomposite that can result in nanofibers using electrospinning technique with improved piezoelectric behavior. The alpha phase is the most common phase of PVDF which is having TGTG^ ${ }^{\wedge}$ conformation (T: trans, G: gauche+, $\mathrm{G}^{\wedge}$ : gauche-) whereas the beta phase is the only piezoelectric phase with all TTTT (trans) conformation. For every alpha PVDF unit cell lattice which consists of two chains in TGTG^, the dipole components cancel each other resulting in zero net dipole moment. But in the case of beta PVDF lattice, unit cell with all trans conformations has its dipoles all aligned in the same direction normal to the chain axis. Thus, all its unit cell consists of dipoles aligned in one direction, and thereby the same is applicable to the molecular dipoles of the beta phase all aligned in one direction generating a large spontaneous polarization for the whole crystal. As a result, it exhibits strong piezoelectric and ferroelectric properties; however, it is difficult to synthesize PVDF with only beta phase contents.
In normal circumstances, the $\alpha$-phase is more easily formed than the $\beta$-phase. Although, the crystal lattice energy of the $\alpha$-phase is slightly higher than that of the $\beta$-phase, direct $\beta$-phase formation from the melt is prohibited due to the high energy of the all-trans conformations. $\beta$-Phase formation can be accomplished via a crystal phase transition from the $\alpha$-phase. The most common technique for obtaining polar $\beta$-PVDF involves mechanical extension (drawing) and electrical poling. Paraelectric phase can be converted into piezoelectric phase by various processes such as crystallization from a polar solution under special conditions-application of high pressure, addition of additive materials to PVDF, etc. A technique used recently is blending with nanofillers such as inorganic materials and electrospinning, such as carbon nanotubes. Carbon nanotube is an allotrope of carbon, which has various types of allotropes, in three dimension, two dimension, one dimension, and zero dimension. 3-D allotrope is common and they are graphite and diamond. The allotropes in the lower dimensions are generally called as carbon nanomaterials. The two-dimensional allotropes of carbon are called graphenes, the onedimensional allotropes are generally called carbon nanotubes, and zero dimension corresponds to fullerenes. Carbon nanotubes can be classified in to two types based on their structure and chiral indices. Depending on the value of chiral indices, there are two types: armchair (with same value of chiral indices) and zigzag structure (with chiral indices value zero). Carbon nanotubes are made up of rolled graphene sheets. Based on the number of rolled-up sheets, carbon nanotubes are classified as single-walled carbon nanotubes where only one thick-walled graphene sheet is wrapped together, double-walled carbon nanotubes where there are two concentrically rolled up graphene sheets, and multiwalled carbon nanotubes (MWCNTs) where there are two or more graphene sheets that roll up each other.

Back again to adding CNTs inside PVDF, the removal of impurities from the carbon nanotubes is done by heating and then adding concentrated sulphuric acid and finally an ultrasonication to maximize the number of carboxylic acid group. Finally, the CNTs are dissolved in the required solvent DMAc which is the solvent for PVDF solution preparation. The randomly oriented well-separated multiwalled CNTs in electrospinning solution become aligned due to the development of straight line at the Taylor cone. Furthermore, the nanotubes are spread along the direction of jet elongation and the resultant nanocomposite fiber is deposited on the collector electrode.

The effect of carbon nanotubes is studied using the addition of 0.05 to $1 \mathrm{wt} . \%$ CNTs with PVDF solution. When $0.05 \%$ CNTs are added to the PVDF solution, a remarkable change in the crystalline structure was observed. Finally, the CNT content was increased, when around $1 \%$ CNTs were added and the increase in interaction between PVDF and CNTs resulted in higher beta content. Thus at the beginning, the addition of MWCNTs in small concentrations even resulted in an abrupt increase in beta phase and thereby piezoelectric performance. But finally after $1 \%$ further addition which did not result in an enormous increase, still there was increase in the beta 
phase content. This is because when MWCNTs are uniformly distributed, the viscosity of the polymer solution increases and they have a stronger interaction between the polymer matrices. More beta phase can be obtained by postdrawing and poling process of the PVDF. Thus in short, when the concentration of MWCNT was increased from 0 to $0.05 \mathrm{wt} . \%$ and finally increased in steps of 0.05 up to $1 \mathrm{wt} . \%$, the beta phase increased abruptly at the beginning up to $2 \%$ increase and then finally further increase in carbon nanotube content will increase the beta phase content in a slower rate till some sort of saturation performance. But the beta phase content can be further increased by mechanical drawing and electrical poling. The performances of the three cases, unpoled undrawn, electrically poled, and mechanically drawn, show same behavior with increased amplitude. All these studies based on the CNT contents are done on generated nanomembranes and not specifically about nanofibers [16, 22].

Another study based on electrospun PVDF nanofiber by varying the MWCNT concentration in the order $0.6 \mathrm{wt} . \%$, $1 \mathrm{wt} . \%$, and $2 \mathrm{wt} . \%$ was done, and the resultant fibers are analyzed using FTIR and spectra clearly shown the enhancement of beta content with increased MWCNT concentration. The infrared band at 837 and $1273 \mathrm{~cm}^{-1}$ which is responsible for beta phase became more prominent with increased MWCNT concentration. The corresponding nanofibers generated using different MWCNT concentrations were analyzed using XRD for checking the crystalline nature. The XRD analysis of the fiber showed a prominent peak at a diffraction peak of 2 theta equals 19.9 degrees, and this corresponds peak to the beta phase, and it is clearly depicted in the figure below. The miller indices corresponding to the beta phase is 110 [44].

(1) Mechanical Properties. The mechanical properties of the electrospun nanofiber for varying MWCNT concentrations are studied. For this, the elongation or strain that happened for a range of stress is studied for different MWCNT concentrations of nanofibers. It is observed that the fiber containing more MWCNTs demonstrated a larger strain which indicates that the carbon nanotubes obviously improved the mechanical properties of electrospun fibers. Also, at the same time, Young's modulus of the electrospun nanofiber is found to be decreasing with increased MWCNT content. It is found that Young's modulus has been decreased from $4.4 \times 10^{-2}$ to $9.1 \times 10^{-3} \mathrm{MPa}$ when the carbon nanotube content increased from 0.6 to $2 \mathrm{wt}$.\% which clearly indicates that the elasticity has been drastically improved by the addition of CNT content $[16,22]$.

(2) Electrical Properties. As we increase the MWCNT content in the composites, the density of the CNT-CNT junction increases up to a particular level. The electrical conductivity of the electrospun nanofibers is found to be increasing linearly with an increase in MWCNT concentration from 0.6 wt.\% to $1.2 \mathrm{wt} . \%$, but after that the further increase in MWCNT content (from 1.2 to $2 \mathrm{wt} . \%$ ) caused saturation state and the conductivity remains constant.
3.3.2. Enhanced Performance of Electrospun Nanofibers by Annealing Process. Approximately 70\% increase in crystallinity of the PVDF-TrFE electrospun nanofibers can be achieved by annealing them at a temperature between Curie temperature and melting temperature (in the paraelectric phase). The increase in crystallinity results in an increase in elastic modulus around 3 times, and piezoelectric effect is found to be enhanced around 55\%. However, both tensile strength and ductility are found to be dropped approximately 1 order of magnitude. Also, some nanoscale cracks are found in the annealed nanofibers, without affecting the strength of it. For example, annealing at a temperature of 130 degrees Celsius resulted in an increase in $90 \%$ piezoelectric constant and $170 \%$ modulus $[19,20]$.

\section{Impact of Alignment of Nanofibers}

The electrospun PVDF nanofibers have a wide range of applications, particularly the aligned nanofibers due to their unique optical, electrical, and mechanical properties. In addition to this, aligned PVDF shows a good response to pressure and a high output voltage. The applications of nanofibers become unlimited only when a set of uniaxial fiber bundles or single nanofibers which are aligned can be developed. Actually, it is a very tough target to be achieved for electrospun PVDF nanofibers due to the bending instability of the polymer jet trajectory. The alignment of the nanofibers is greatly influenced by the collector electrode structure and some other key experimental parameters such as flow rate and PVDF solution concentration. Another simple and successful method for the production of spinning sheets with aligned parallel fibers is the use of a circular drum where copper wires are evenly spaced. Actually, this method is unique and robust for the creation of aligned nanofibers. Thus, aligned electrospun nanofibers are of great interest and our studies mainly focus on the improvement of alignment with electrospinning technique $[45,46]$.

\section{Brief Applications Based on PVDF Nanofibers}

Nanotechnology has a wide range of applications in almost all fields of life including medicine, textile industry, engineering field, and cosmetics. The special applications of electrospun nanofibers include drug delivery, wound dressing, biosensors, lithium-ion batteries, scaffold for tissue engineering, medical implants, photocatalytic degradation, and water proof garments. Nanofibers have a special role in the purification of water by filtering process. Some important applications of PVDF electrospun nanofibers include its use in energy scavenging applications, in application in tissue engineering, in nanopower generator, as a separation medium for oil water separation, and in electromechanical sensors, as a piezoelectric transducer, etc.

Some important studies based on the applications of nanofiber as a nanoelectromechanical sensor revealed that a single suspended nanofiber has a piezoelectric coefficient $\left(D_{33}\right)$ of $\sim 58.77 \mathrm{pm} / \mathrm{V}$. They were able to incorporate the single nanofiber onto a microelectromechanical system 
(MEMS) and demonstrate its ability to sense dynamic flow generated by oscillatory dipole stimulus. Another important application that utilizes piezoelectric behavior is in power generation. A study by Chang et al. proposed that a single nanofiber has an energy conversion efficiency of $12.5 \%$ going as high as $21.8 \%$ which is much higher than the energy conversion efficiency of a nanothin film which has about $1.3 \%$ average [47].

A medical application of electrospun nanofiber that utilizes both aligned and random orientation is in $3 \mathrm{D}$ bifunctionalized nerve conduits. In this study, they developed a nerve conduit with the inner surface incorporated with aligned nanofibers which is able to enhance the proliferation of neural cells. Since the mechanical strength of aligned fibers are very weak, the central part of the tube was double coated with randomly oriented fibers which improve the mechanical strength of the aligned fiber. This study developed a novel electrospinning technique where a fiber mat containing both aligned and random fibers is synthesized. The studies are based on PU and PLGA nanofibers, and the scope of PVDF in this field is in a progressing stage. Further analysis and studies are required in this field for utilizing PVDF in nerve conduits $[48,49]$.

\section{Conclusion}

In this review, a detailed study of utilization of electrospinning technique for generating random and aligned PVDF nanofibers is presented. The optimum conditions of experimental parameters required for the achievement of aligned nanofibers are studied. At the same time, the piezoelectric property of the aligned PVDF nanofiber and the methods for enhancing the piezoelectric behavior are also studied. Electrospinning technique using PVDF is a promising tool for both aligned and random nanofibers, and the characterization techniques used for the analyses of the fabricated fibers are also discussed. A survey on the relevant applications in these fields is also done which is greatly helpful for our future experimental studies and developments.

\section{Conflicts of Interest}

The mentioned received funding did not lead to any conflict of interests regarding the publication of this manuscript.

\section{Acknowledgments}

The authors are grateful to Kuwait College of Science and Technology for both logistic and financial support provided for the present and advanced studies involved in this research field.

\section{References}

[1] M. Mirjalili and S. Zohoori, "Review for application of electrospinning and electrospun nanofibers technology in textile industry," Journal of Nanostructure in Chemistry, vol. 6, no. 3, pp. 207-213, 2016.

[2] F. Mokhtari, M. Latifi, and M. Shamshirsaz, "Electrospinning/ electrospray of polyvinylidene fluoride (PVDF): piezoelectric nanofibers," The Journal of The Textile Institute, vol. 107, pp. 1-19, 2015.

[3] J. Zhao, N. Si, L. Xu, X. Tang, Y. Song, and Z. Sun, "Experimental and theoretical study on the electrospinning nanoporous fibers process," Materials Chemistry and Physics, vol. 170, pp. 294-302, 2016.

[4] Z.-M. Huang, Y.-Z. Zhang, M. Kotaki, and S. Ramakrishna, "A review on polymer nanofibers by electrospinning and their applications in nanocomposites," Composites Science and Technology, vol. 63, no. 15, pp. 2223-2253, 2003.

[5] D. Sengupta, A. G. P. Kottapalli, S. H. Chen et al., "Characterization of single polyvinylidene fluoride (PVDF) nanofiber for flow sensing applications," AIP Advances, vol. 7, no. 10, article 105205, 2017.

[6] L. Yu, S. Wang, Y. Li et al., "Piezoelectric performance of aligned PVDF nanofibers fabricated by electrospinning and mechanical spinning," in 2013 13th IEEE International Conference on Nanotechnology (IEEE-NANO 2013), Beijing, China, 2013.

[7] M.-G. Kang, W.-S. Jung, C.-Y. Kang, and S.-J. Yoon, "Recent progress on PZT based piezoelectric energy harvesting technologies," Actuators, vol. 5, no. 1, 2016.

[8] X.-f. Liu, C.-x. Xiong, H.-j. Sun, L.-j. Dong, R. li, and Y. Liu, "Piezoelectric and dielectric properties of PZT/PVC and graphite doped with PZT/PVC composites," Materials Science and Engineering: B, vol. 127, no. 2-3, pp. 261-266, 2006.

[9] A. Datta, Y. S. Choi, E. Chalmers, C. Ou, and S. Kar-Narayan, "Piezoelectric Nylon 11 nanowire arrays grown by template wetting for vibrational energy harvesting applications," Advanced Functional Materials, vol. 27, no. 2, article 1604262, 2017.

[10] L. Persano, C. Dagdeviren, Y. Su et al., "High performance piezoelectric devices based on aligned arrays of nanofibers of poly(vinylidenefluoride cotrifluoroethylene)," Nature Communications, vol. 4, no. 1, article 2639, 2013.

[11] J. Chang, M. Dommer, C. Chang, and L. Lin, "Piezoelectric nanofibers for energy scavenging applications," Nano Energy, vol. 1, no. 3, pp. 356-371, 2012.

[12] E. Stojanovska, E. Canbay, E. S. Pampal et al., "A review on non-electro nanofibre spinning techniques," RSC Advances, vol. 6, no. 87, pp. 83783-83801, 2016.

[13] G. Zhu, L. Y. Zhao, L. T. Zhu, X. Y. Deng, and W. L. Chen, "Effect of experimental parameters on nanofiber diameter from electrospinning with wire electrodes," IOP Conference Series: Materials Science and Engineering, vol. 230, article 012043, 2017.

[14] Z. Li and C. Wang, "One dimensional nanostructures electrospinning technique and unique nanofibers," Journal of Physical Chemistry C, vol. 117, pp. 11791-11799, 2013.

[15] A. E. Senador, M. T. Shaw, and P. T. Mather, "Electrospinning of polymeric nanofibers: analysis of jet formation," Materials Research Society Symposia Proceedings, vol. 661, 2000.

[16] Y. Ahn, J. Y. Lim, S. M. Hong et al., "Enhanced piezoelectric properties of electrospun poly (vinylidene fluoride)/multiwalled carbon nanotube composites due to high $\beta$ phase formation in poly (vinylidene fluoride)," Journal of Physical Chemistry C, vol. 117, no. 22, pp. 11791-11799, 2013.

[17] E. Zampetti, A. Bearzotti, and A. Macagnano, "Flexible piezoelectric transducer based on electrospun PVDF nanofibers for sensing applications," Procedia Engineering, vol. 87, pp. 1509$1512,2014$. 
[18] C. Baur, J. R. DiMaio, E. McAllister et al., "Enhanced piezoelectric performance from carbon fluoropolymer nanocomposites," Journal of Applied Physics, vol. 112, no. 12, article 124104, 2012.

[19] X. Shi, W. Zhou, D. Ma et al., "Electrospinning of nanofibers and their applications for energy devices," Journal of Nanomaterials, vol. 2015, Article ID 140716, 20 pages, 2015.

[20] Z. H. Liu, C. T. Pan, L. W. Lin, J. C. Huang, and Z. Y. Ou, "Direct write PVDF nonwoven fiber fabric energy harvesters via the hollow cylindrical near-field electrospinning process," Smart Materials and Structures, vol. 23, no. 2, article 025003, 2014.

[21] W. E. Teo and S. Ramakrishna, "A review on electrospinning design and nanofibre assemblies," Nanotechnology, vol. 17, no. 14, pp. R89-R106, 2006.

[22] A. Yousefi, "Hybrid polyvinylidene fluoride/nanoclay/ MWCNT nanocomposites: PVDF crystalline transformation," Iranian Polymer Journal, vol. 20, no. 9, pp. 725-733, 2011.

[23] X. Wang, J. Yu, G. Sun, and B. Ding, "Electrospun nanofibrous materials: a versatile medium for effective oil/water separation," Materials Today, vol. 19, no. 7, pp. 403-414, 2016.

[24] J. Song, G. Zhao, B. Li, and J. Wang, "Design optimization of PVDF-based piezoelectric energy harvesters," Heliyon, vol. 3, no. 9, article e00377, 2017.

[25] M. Baniasadi, Z. Xu, S. Hong, M. Naraghi, and M. MinaryJolandan, "Thermo-electromechanical behavior of piezoelectric nanofibers," ACS Applied Materials \& Interfaces, vol. 8, no. 4, pp. 2540-2551, 2016.

[26] M. Baniasadi, Z. Xu, J. Cai et al., "Correlation of annealing temperature, morphology, and electro-mechanical properties of electrospun piezoelectric nanofibers," Polymer, vol. 127, no. 3, pp. 192-202, 2017.

[27] B. J. Love, D. Leo, and D. Inman, Manufacturing of Poly (Vinylidene Fluoride) and Evaluation of Its Mechanical Properties, vtechworks, 2002.

[28] J. Bernal-Martínez, A. Seseña-Rubfiaro, R. Godínez-Fernández, and A. Aguilar-Elguezabal, "Electrodes made of multiwall carbon nanotubes on PVDF-filters have low electrical resistance and are able to record electrocardiograms in humans," Microelectronic Engineering, vol. 166, pp. 10-14, 2016.

[29] S. M. Hosseini and A. A. Yousefi, "Electrospun PVDF/ MWCNT/OMMT hybrid nanocomposites: preparation and characterization," Iranian Polymer Journal, vol. 26, no. 5, pp. 331-339, 2017.

[30] A. A. A. I. Ramadan, Physico-Mechanical Properties of New Electrospun Polyvinylidene Fluoride Nanocomposites, [M.S. Thesis], Material Science and Technology, 2015.

[31] C. M. Wu and M. H. Chou, "Polymorphism, piezoelectricity and sound absorption of electrospun PVDF membranes with and without carbon nanotubes," Composites Science and Technology, vol. 127, pp. 127-133, 2016.

[32] C. L. Yang, Z. H. Li, W. J. Li et al., "Batwing-like polymer membrane consisting of PMMA-grafted electrospun PVDF$\mathrm{SiO}_{2}$ nanocomposite fibers for lithium-ion batteries," Journal of Membrane Science, vol. 495, pp. 341-350, 2015.

[33] Y. Ting, H. Gunawan, A. Sugondo, and C. W. Chiu, “A new approach of polyvinylidene fluoride (PVDF) poling method for higher electric response," Ferroelectrics, vol. 446, no. 1, pp. 28-38, 2013.

[34] E. S. Cozza, O. Monticelli, E. Marsano, and P. Cebe, “On the electrospinning of PVDF: influence of the experimental conditions on the nanofiber properties," Polymer International, vol. 62, no. 1, pp. 41-48, 2013.

[35] K. Ke, P. Potschke, D. Jehnichen, D. Fischer, and B. Voit, "Achieving $\beta$-phase poly (vinylidene fluoride) from melt cooling: effect of surface functionalized carbon nanotubes," Polymer, vol. 55, no. 2, pp. 611-619, 2014.

[36] F. Yalcinkaya, "Preparation of various nanofiber layers using wire electrospinning system," Arabian Journal of Chemistry, 2016.

[37] S. Kripotou, S. Sovatzoglou, C. Pandis et al., "Effects of CNT inclusions on structure and dielectric properties of PVDF/ CNT nanocomposites," Phase Transitions, vol. 89, no. 7-8, pp. 717-730, 2016.

[38] K. Yoon and A. Kelarakis, "Nanoclay-directed structure and morphology in PVDF electrospun membranes," Journal of Nanomaterials, vol. 2014, Article ID 367671, 7 pages, 2014.

[39] K. Arayanarakul, N. Choktaweesap, D. Aht-ong, C. Meechaisue, and P. Supaphol, "Effects of poly (ethylene glycol), inorganic salt, sodium dodecyl sulfate, and solvent system on electrospinning of poly (ethylene oxide)," Macromolecular Materials and Engineering, vol. 291, no. 6, pp. 581-591, 2006.

[40] M. Nasir, H. Matsumoto, M. Minagawa, A. Tanioka, T. Danno, and H. Horibe, "Preparation of Porous PVDF Nanofiber from PVDF/PVP Blend by Electrospray Deposition," Polymer Journal, vol. 39, no. 10, pp. 1060-1064, 2007.

[41] A. Gheibi, R. Bagherzadeh, A. A. Merati, and M. Latifi, "Electrical power generation from piezoelectric electrospun nanofibers membranes: electrospinning parameters optimization and effect of membranes thickness on output electrical voltage," Journal of Polymer Research, vol. 21, no. 11, p. 571, 2014.

[42] A. Ledoux, Theory of Piezoelectric Materials and Their Applications in Civil Engineering, Thesis Master of Engineering in Civil and Environmental Engineering MIT, 2011.

[43] D. Dhakras, V. Borkar, S. Ogale, and J. Jog, "Enhanced piezoresponse of electrospun PVDF mats with a touch of nickel chloridehexahydrate salt," Nanoscale, vol.4, no. 3,pp.752-756, 2012.

[44] S.-H. Wang, Y. Wan, B. Sun, L.-Z. Liu, and W. Xu, "Mechanical and electrical properties of electrospun PVDF/MWCNT ultrafine fibers using rotating collector," Nanoscale Research Letters, vol. 9, no. 1, p. 522, 2014.

[45] L. Persano, C. Dagdeviren, Y. Su et al., "High performance piezoelectric devices based on aligned arrays of nanofibers of poly[(vinylidenefluoride-co-trifluoroethylene)]," Nature Communications, vol. 4, no. 1, article 1633, 2013.

[46] P. Katta, M. Alessandro, R. D. Ramsier, and G. G. Chase, "Continuous electrospinning of aligned polymer nanofibers onto a wire drum collector," Nano Letters, vol. 4, no. 11, pp. 2215-2218, 2004.

[47] C. Chang, K. Limkrailassiri, and L. Lin, "Continuous near-field electrospinning for large area deposition of orderly nanofiber patterns," Applied Physics Letters, vol. 93, no. 12, article 123111, 2008.

[48] C. Huang, Y. Ouyang, H. Niu et al., "Nerve guidance conduits from aligned nanofibers: improvement of nerve regeneration through longitudinal nanogrooves on a fiber surface," ACS Applied Materials \& Interfaces, vol. 7, no. 13, pp. 7189-7196, 2015.

[49] Z. Li and C. Wang, "Effects of working parameters on electrospinning," in One Dimensional NanostructuresSpringerBriefs in Materials. 


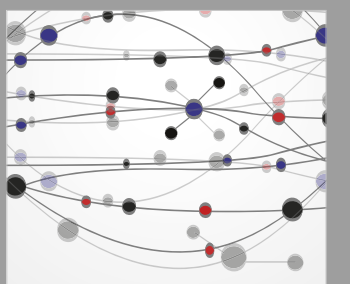

The Scientific World Journal
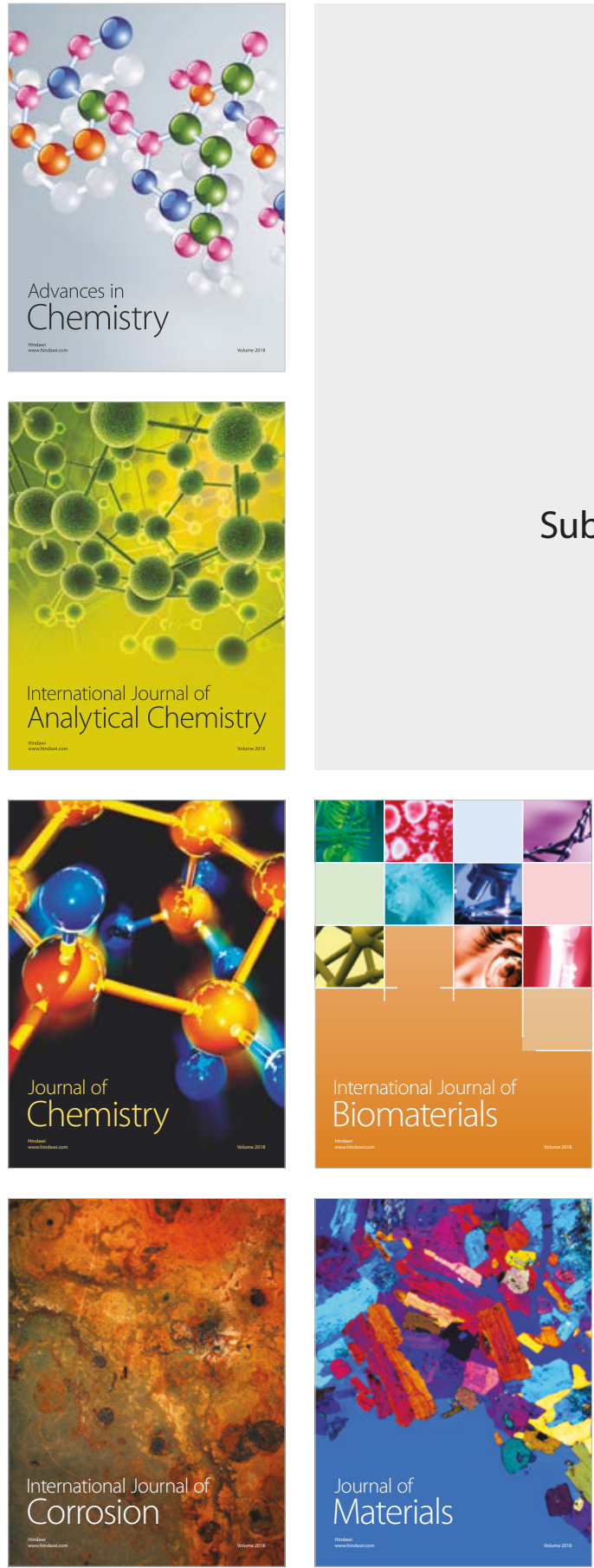

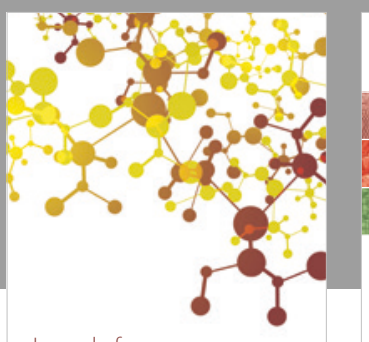

Journal of

Applied Chemistry
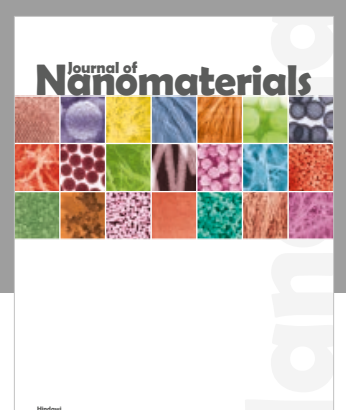

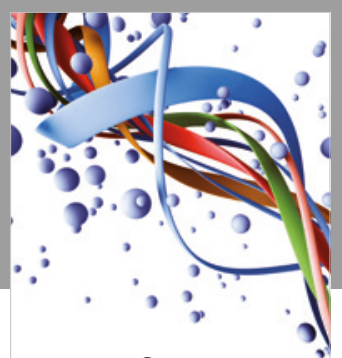

Scientifica

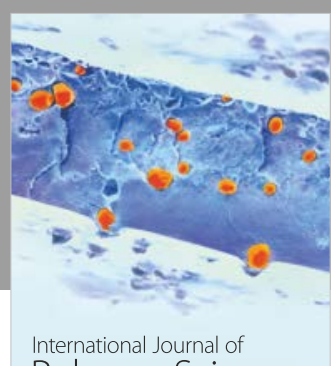

Polymer Science

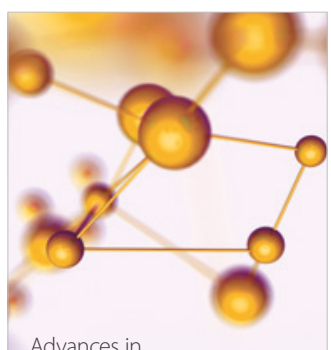

Physical Chemistry
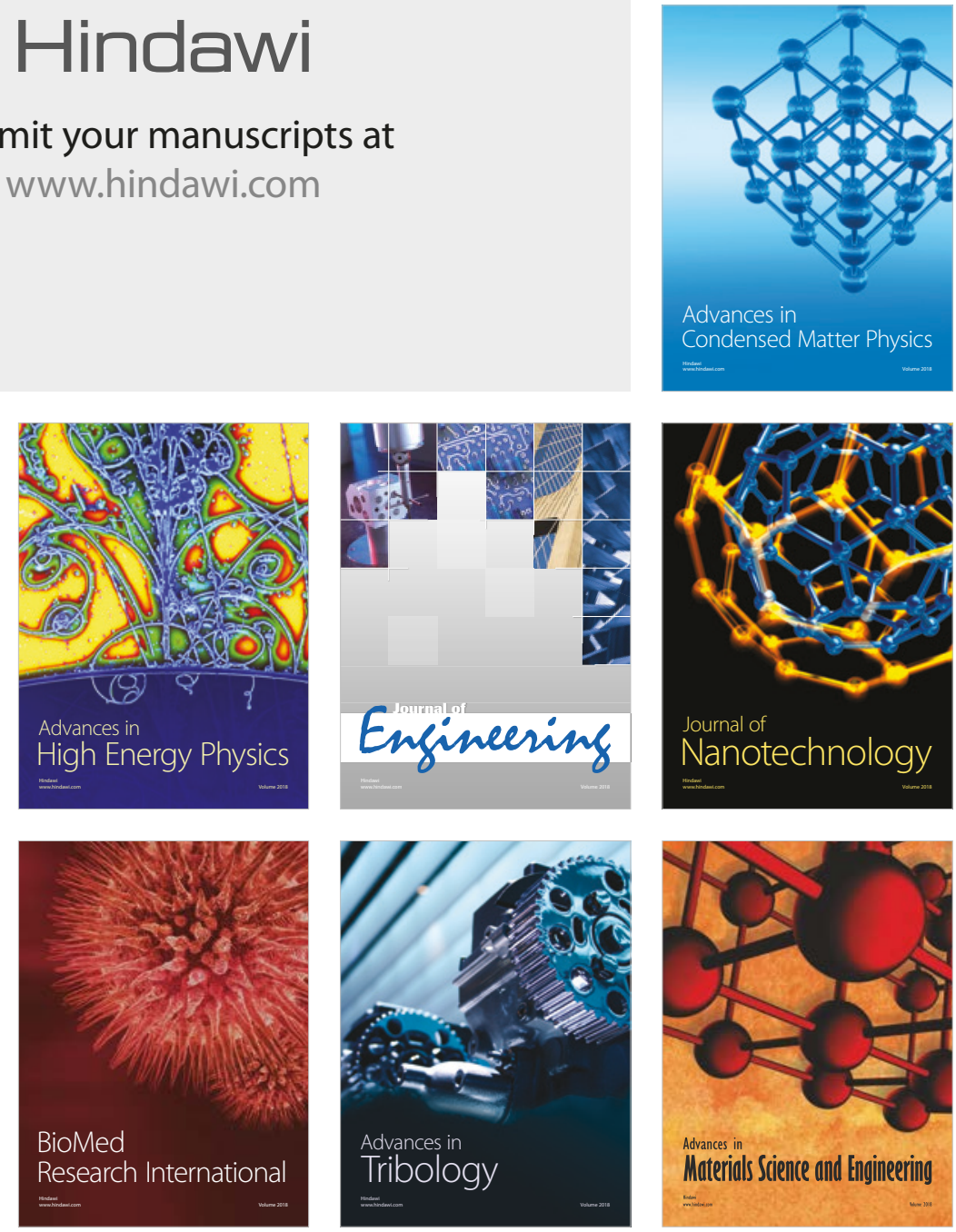